\title{
An Internal Model Controller for Three-Phase APF Based on LS-Extreme Learning Machine
}

\author{
Zhenhua Shao*, Tianxiang Chen, Li-an Chen and Hong Tian
}

Department of Electrical Engineering and Automation Xiamen University of Technology, Xiamen Fujian, 361024, China

\begin{abstract}
Aiming at the problem that the three-phase APF's dynamic model is a multi-variable, nonlinear and strong coupling system, an internal model controller for three-phase APF based on LS-Extreme Learning Machine is studied in this paper. As a novel single hidden layer feed-forward neural networks, extreme learning machine (ELM) has several advantages: simple net structural, fast learning speed, good generalization performance and so on. In order to improve the controller's dynamic responses, a least squares extreme learning machine for internal model control is proposed. A least squares ELM regression (LS-ELMR) model for the three-phase APFS on-line monitoring was built from external factors with in-out datum. Moreover, the relative stable error is presented to evaluate the system performance and the features for the internal model control system based on extreme learning machine, neural network, kernel ridge regress and support vector machine. The experimental results show that the LS-internal model control system based on extreme learning machine has good dynamic performance and strong filtering result.
\end{abstract}

Keywords: Extreme learning machine (ELM), internal model control (IMC), Shunt active power filter (SAPF), Least squares (LS), Robustness.

\section{INTRODUCTION}

Nowadays, with the rapid development of power electronic equipments and wide proliferations of nonlinear loads, the increasing harmonic currents in power grid and a lower power factor are responsible for the additional power losses and the risk of power equipment's damage [1]. Since the power system is often influenced by many uncertainties such as nonlinear, non-stationary, complexity and randomness etc., Shunt Active Power Filters (SAPF) are regarded as the effective way to deal with the problem of reactive power compensation and harmonic suppression due to their high switching frequencies and employing digital signal processors. Moreover, there is no doubt that the Pulse Width Modulation (PWM) inverter plays an important role in digital current control owing to the rapid progress of microprocessor technology and fast dynamic response [2-4]. The current control is the very important part of the overall control system.

On the other hand, dynamic performance of APF is mainly dependent on advanced data control technique and power electronic technology, especially the accuracy and stability of APF's mathematics models. A lot of scholars pay much more attentions on the study of three-phase APF current control [5-8]. The commonly used control methods at present can be mainly divided into several kinds: PI control, deadbeat control, variable-structure control, fuzzy logical control, predict control, ANN control and so on. Considering the nonlinear character of APF system, especially the randomized complexity and non-stationary interconnected

*Address correspondence to this author at the Department of Electrical Engineering and Automation Xiamen University of Technology, Xiamen Fujian, 361024, China; E-mail: Szh4h@163.com power system, the effective inverse system mathematics models of APFs are lacking.

Since the IMC strategy had been proposed by Carcia and Morari in the 1980's, the IMC has been considered to have an effective function on the control of uncertain and pure delay systems due to its simple structure, strong tracking robustness, good anti-interference effect etc. Since the controlled plant usually has a nonlinear characteristic, the nonlinear IMC based on intelligent learning parameter estimation model has been successfully applied in practical engineering. Moreover, the nonlinear IMC based on neural network $(\mathrm{NN})$ has been used more and more widely, and the typical algorithm is the feedback neural network. However, it is well known that the parameters of traditional feedforward neural network all need to be adjusted and the parameters of different layers will influence each other. On the other hand, as one of the most common algorithms in parameters adjustment, the Gradient Descent Method (GDM) has two inherent defects of locally minimum and slowly convergence speed in the usually adoptive BP network. So it is necessary to find a novel parameter estimation method for nonlinear IMC system.

As a novel single hidden layer feed-forward neutral network, ELM was proposed by professor G.-B. Huang in 2004. Compared with the traditional method, ELM greatly improves the learning rate of feed-forward neural networks; meanwhile ELM also has good generalization ability. Furthermore the neural weight can be obtained by MoorePenrose generalized inverse calculation, and shortcomings of GDM can be avoided: such as locally minimum, large iteration number, the difficult selection of suitable performance parameters and learning rate etc. ELM is gaining more and more attention due to its high learning rate and excellent 
neutral generalization ability. And ELM has become one of the most promising methods of learning algorithms. Some successful applications of ELM can be found in many research fields [9-11]. In this paper, the internal model and the inverse model of IMC are fitted in ELM. And the experimental results show that compared with the classical learning algorithms in neural networks, e.g. RBF networks, ELM can achieve better performance in much shorter learning time. Furthermore, the LS-ELM has a better filtering result.

With the wide application of nonlinear industrial loads, especially the power electronic equipment, the power system harmonic pollution problems become increasingly serious, which have led to the deterioration of power quality and have done great harm to the safe and economic operation of power system. As an effective way of dynamic harmonic suppression and reactive compensation, APF's have been widely applied in power supply system and industrial system[1-3]. Moreover, the topology, the design optimization of output filter and snobbier, the control method, and the implementation of APF play important roles in the effects of harmonics elimination. In most high performance industrial systems, such as frequency control system and three-phases $\mathrm{AC} / \mathrm{DC}$ converters.

\section{EXTREME LEARING MACHINE}

Given a training set (xi, yi),

$x_{i}=\left[x_{i 1}, x_{i 2}, \ldots, x_{i n}\right]^{T} \in R^{n}$,

$y_{i}=\left[t_{i 1}, t_{i 2}, \ldots, t_{i m}\right]^{T} \in R^{m}, \quad \mathrm{i}=1,2, \ldots, \mathrm{N}$.

If a standard single layer feed-forward neural network with $\mathrm{N}$ hidden nodes and $\mathrm{g}(\mathrm{x})$ is the activation function, then the mathematical model can be expressed as follows:

$$
\sum_{i=1}^{L} \beta_{i} g_{i}\left(x_{j}\right)=\sum_{i=1}^{L} \beta_{i} g\left(w_{i} \cdot x_{j}+b_{i}\right)=t_{j}
$$

Where $w i$ is the weight vector connecting inputs and the $i$ th hidden neurons, $\beta_{i}=\left[\beta_{i 1} \beta_{i 2}, \ldots, \beta_{i m}\right]^{T}$ is the weight vector connecting outputs and the $i$ th hidden neurons, $b i$ is the $i$ th hidden neurons, $i=1,2, \ldots \ldots, \mathrm{N}$.

The above $\mathrm{N}$ equations can be written in matrix form as follows:

$\mathrm{H} \beta=\mathrm{Y}$

Where

$$
\begin{aligned}
& \mathrm{H}\left(w_{1}, \ldots, w_{L}, b_{1}, \ldots, b_{L}, x_{1}, \ldots, x_{N}\right) \\
& =\left[\begin{array}{ccc}
g\left(w_{1} x_{1}+b_{1}\right) & \cdots & g\left(w_{L} x_{1}+b_{L}\right) \\
\vdots & \ldots & \vdots \\
g\left(w_{1} x_{N}+b_{1}\right) & \cdots & g\left(w_{L} x_{N}+b_{L}\right)
\end{array}\right]_{N \times L} \\
& \qquad \mathrm{Y}=\left[\begin{array}{c}
y_{1}^{T} \\
\vdots \\
y_{N}^{T}
\end{array}\right]_{N \times m}
\end{aligned}
$$

According to the definition in [12], $\mathrm{H}$ is the output matrix of the hidden neurons. Since $\mathrm{L} \ll \mathrm{N}$ and $\mathrm{H}$ is not a square matrix, the inverse matrix $\mathrm{H}-1$ of matrix $\mathrm{H}$ exists. Given any input weights $w$ and bias $b$, and H-1 can be calculated by Moore-Penrose generalized inverse theory. Then

$\beta=H^{-1} Y$

In this way, the network parameters of ELM can be obtained by equations (3) and (4). Furthermore the network of ELM can be established as in Fig. (1)

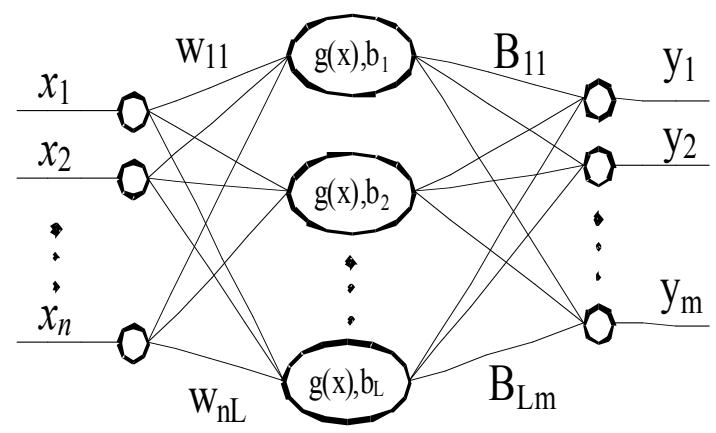

Fig. (1). The network of ELM.

As is shown in Fig. (1), the network parameters of ELM (the number of hidden nodes L, activation function $g(x)$, given any parameters $w$ and $b$ ) will be set once so there is no further parameters adjustment. As the explanation of the authors [13], the minimum training error, the smallest norm of weights and best generalization performance can be obtained by the ELM algorithm.

Lemma.1 Given arbitrary small number $\varepsilon$ and infinitely derivable function $g(x)$, there must exist $L<<N(N$ is the sample size); given any $w$ and $b,\|H \beta-T\|<\varepsilon$.

Theorem.1 Given an infinitely derivable function $g(x)$, the mathematical expression (2) of ELM is also infinitely derivable.

According to the derivation process of ELM, the concrete values of net parameters $w, b, \beta, L$ of ELM are confirmed as soon as the network learning of ELM is completed. Given an infinitely derivable function $g(x)$, there are four arithmetic $\begin{array}{ll}\text { operations, } & g^{\prime}(w \cdot x+b) \beta=g^{\prime}(w \cdot x+b) \beta+g(w \cdot x+b) \beta^{\prime} \\ = & (g(w \cdot x+b) \beta)^{\prime}\end{array}$

Then $(g(x) \beta)$ is an infinitely derivable function, that is, the ELM algorithm is also infinitely derivable.

\section{CONSTRUCTION AND MATHEMATICAL MODEL OF THREE-PHASE SAPF}

\subsection{The Principle of SAPF and Main Circuit}

The main circuit of three-phase SAPF is shown in Fig. (2). The SAPF system is designed to compensate harmonics, unbalance and reactive power. As can be seen in Fig. (2), shunt active power filter is connected in parallel with the nonlinear load. By injecting equal but opposite harmonic 


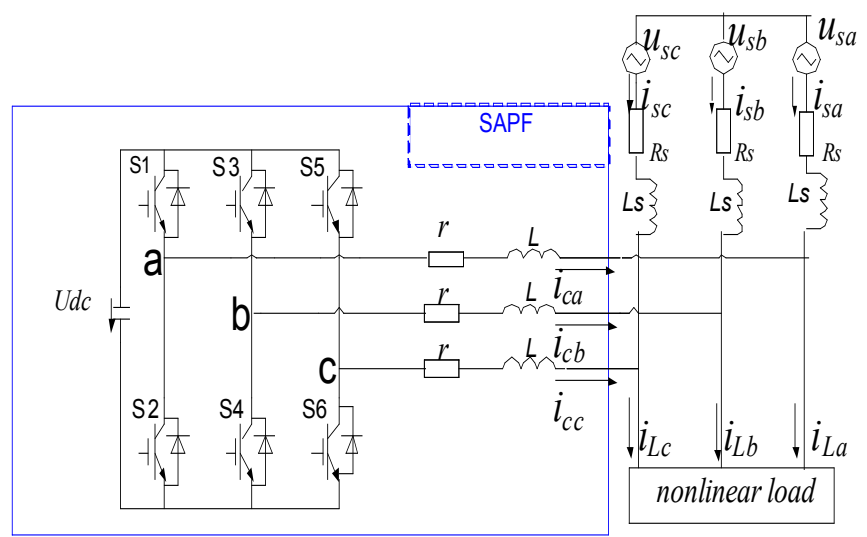

Fig. (2). Topology of three-phase SAPF.

current components at the point of connection, the desired sinusoidal active current can be obtained. Where $i a, i b, i c$ are the compensation currents, isa,isb,isc are the three-phase source currents, $V_{s} a, V s b, V s c$ are the three-phase source voltages, and ila,ilb,ilc are the load currents. $L s$ and $R s$ are the equivalent source inductance and resistance respectively.

\subsection{The Dynamic Model of Three-Phase SAPF}

According to the reference [14], the dynamic model of error signals can be obtained as follows:

$$
\left\{\begin{array}{c}
\frac{d \Delta i_{d}}{d t}=-\frac{r+2 R_{s}}{L+L_{s}} \Delta i_{d}+\omega \Delta i_{q}+\frac{S_{a}}{L+L_{s}} \Delta V_{d c}+w_{1} \\
\frac{d \Delta i_{q}}{d t}=-\omega \Delta i_{d}-\frac{r+2 R_{s}}{L+L_{s}} \Delta i_{q}+\frac{S_{b}}{L+L_{s}} \Delta V_{d c}+w_{2} \\
\frac{d \Delta i_{0}}{d t}=\left(\frac{4 r+2 R_{0}+R_{s}}{4 L+3 L_{0}+L_{s}}\right) \Delta i_{0}+\frac{S_{c}}{4 L+3 L_{0}+L_{s}} \Delta V_{d c}+w_{3} \\
\frac{d \Delta V_{d c}}{d t}=-\frac{1}{C}\left(S_{a} \Delta i_{d}+S_{b} \Delta i_{q}+S_{c} \Delta i_{0}+\frac{\Delta V_{d c}}{R}\right)+w_{4}
\end{array}\right.
$$

Where wi $(i=1,2,3,4)$ are the external disturbances, $\mathrm{Sa}, \mathrm{Sb}, \mathrm{Sc}$ are the switching functions under $d-q-0$ coordinate axis, $\Delta \mathrm{Vdc}$ is the $\mathrm{DC}$ capacitor voltage variation, $\Delta i d, \Delta i q, \Delta i 0$ are the current variations under $d-q-0$ coordinate axis. If state variables $[\Delta i d, \Delta i q, \Delta i 0, \Delta \mathrm{V} d c] T=[x 1, x 2, x 3, x 4] T$, input variables $[\mathrm{Sa}, \mathrm{Sb}, \mathrm{Sc}] \mathrm{T}=[\mathrm{u} 1, \mathrm{u} 2, \mathrm{u} 3] \mathrm{T}$, and output variables $[\Delta i d$, $\Delta i q, \Delta i 0] \mathrm{T}=[\mathrm{y} 1, \mathrm{y} 2, \mathrm{y} 3]$, the dynamic errors equation of threephase SAPF can be obtained.

\section{INTERNAL MODEL CONTROL BASED ON ELM}

As is shown in Fig. (3) the IMC of nonlinear system consists of four parts: plant $(\mathrm{P})$, internal model $(\mathrm{M})$, controller (C) and robust filter $(\mathrm{F})$.

\subsection{Internal Model Based on LS-ELM}

Considering that the controlled plant $\mathrm{P}$ is the single-input and single-output $\mathrm{N}$-multi system, with $u(k), u(k-1), \ldots, u(k$ $n+1)$ as input variable, $y(k), y(k-1), \ldots, y(k-n+1)$ as output variable and $y(k+1)$ as the system output, then the ARMA (autoregressive moving average model) can be described as follows:

$y(k+1)=P\left(w_{k}, u(k)\right)$

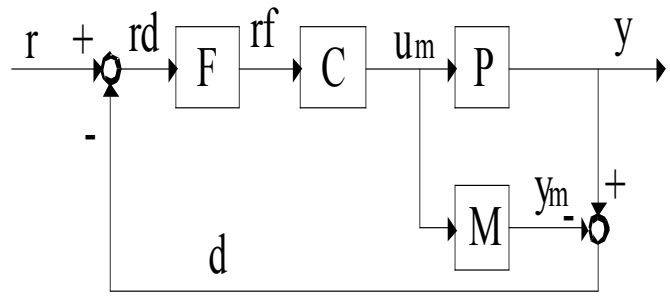

Fig. (3). IMC of nonlinear system.

where $w k=[y(k), \ldots, y(k-n+1), u(k-1), \ldots, u(k-n+1)]$, input $u(k) \quad R$, output $y(k) \quad R, \quad P: R n \times R n \rightarrow R$, and $P(0,0)=0$.

According to lemma 1, the inter model can be obtained with little modeling error $\sigma k$ :

$y(k+1)=y_{m}(k+1)+\sigma_{k}=\operatorname{elm}\left(w_{k}, u(k)\right)+\sigma_{k}$

where $|\sigma \mathrm{k}|<\sigma, \quad \sigma$ is arbitrary small positive number.

According to theorem 1, ELM is an infinitely divisible algorithm. If there is a deviation in $u(k)$, that is $u m(k)=u(k)$ $\Delta$. Based on Taylor series expansion, the approximate value of equation (7) can be as follows:

$\operatorname{elm}\left(w_{k}, u_{m}(k)\right)=\operatorname{elm}\left(w_{k}, u(k)\right)+\frac{\partial\left(e \operatorname{lm}\left(w_{k}, u(k)\right)\right)}{\partial(u(k))} \Delta$

According to equation (11), the convergence rate and convergence precision of ELM is determined. In order to have a good controlled result, a nonlinear LS function optimization method algorithm L-M method is needed [15].

Where $[$ elm, $]=\min -\operatorname{LM}\left({ }^{e l m}\left(w_{k}, u_{m}(k)\right.\right.$.

elm(w $\left.w_{k}, u(k)\right)$;elm(0); beta;u;v;var;eps)

\subsection{Inverse Model Based on ELM}

If the nonlinear system is inverse, the inverse system $\mathrm{C}$ of the control plant can be obtained. If there is an error $\varepsilon \mathrm{k}$, the inverse system with ELM can be described as:

$u(k)=u_{m}(k)+\varepsilon_{k}=\operatorname{elmi}\left(v_{k}, y(k+1)\right)+\varepsilon_{k}$

Where $v k=[y(k), \ldots, y(k-n+1), u(k-1), \ldots, u(k-n+1)]$, and $|\varepsilon k|<\varepsilon$, $\varepsilon$ is arbitrary small positive number.

\section{ANALYSIS OF STEADY-STATE ERROR}

As a typical nonlinear system, the accurate mathematics model of three-phase APF system can be not obtained, so the steady-state error of nonlinear IMC system should be considered. As the Fig. (3), the steady-state error $e(k)=y(k)-r(k$ 1),

then $d(k)=y(k)-y m(k)=-\sigma k$

and $r d(k)=r(k)+\sigma k$

If there is a linear filter $\mathrm{F}$,

then $u_{m}(k)=\operatorname{elmi}\left(v_{k}, r d(k) F\right)$

If there is a modeling error $\varepsilon k$, then $u(k)=u m(k)+\varepsilon k$. 
According to equation (7), $y(k+1)=y m(k+1)+\sigma k=e l m(w k, u m(k)+\varepsilon k)+\sigma k$

Based on Taylor series expansion at the point of $u(k)$, the equation (13) can be expressed as:

$y(k+1)=\operatorname{elm}\left(w_{k}, u_{m}(k)\right)+\frac{\partial\left(e \operatorname{lm}\left(w_{k}, u_{m}(k)\right)\right)}{\partial u_{m}(k)} \varepsilon_{k}+\sigma_{k}$

If $\frac{\partial\left(e \operatorname{lm}\left(w_{k}, u_{m}(k)\right)\right)}{\partial u_{m}(k)}=M$, where $\mathrm{M}$ bounded real number, combining with equations (12) and (14),

$y(k+1)=\operatorname{elm}(w k, \operatorname{elmi}(v k, r d(k) F))+M \varepsilon k+\sigma k$

In that the controlled system is inverse, according to equations (7) and (10):

$y(k+1)=r d(k) F+M \varepsilon_{k}+\sigma_{k}$

As $\mathrm{F}$ is a one-step linear filter, then

$F=\frac{1-\alpha}{1-\alpha z^{-1}}$

Where $0<\alpha<1$.

According to equations (16) and (17), if $|\sigma \mathrm{k}|<\sigma$ and $|\varepsilon k|<\varepsilon$, then:

$y(k+1)-r(k)=\alpha(y(k)-r(k))+M(1-\alpha) \varepsilon+(2-\alpha) \sigma$

As $r(k)=r(k-1)+\Delta r$, where $\Delta r$ is real number, then

$e(k+1)=\alpha e(k)-\alpha \Delta r+M(1-\alpha) \varepsilon+(2-\alpha) \sigma$

If the Lyapunov function of the IMC system $V(k)=e 2(k)$, and $A=M(1-\alpha) \varepsilon+(2-\alpha) \sigma$, then

$V(k+1)-V(k)=e^{2}(k+1)-e^{2}(k)$

$=\left(\alpha^{2}-1\right) e^{2}(k)+2 \alpha(A-\alpha \Delta r) e(k)+(A-\alpha \Delta r)^{2}$

According to the equation (20), characteristic root criterion $\Delta=4(A-\alpha \Delta r) 2>0$,

if $(A-\Delta r \alpha)>0$, then

$e(k)>\frac{A-\Delta r \alpha}{1+\alpha}$

Then function $(20)<0$, and the IMC system is a stable system.

if $(A-\Delta r \alpha)<0$, then

$e(k)<\frac{A-\Delta r \alpha}{1+\alpha}$

Then function $(20)<0$, and the IMC system is also a stable system.
When $0<\alpha<1$, the series $e(k)$ is convergent and the state error is

$e(\infty)=\lim _{k \rightarrow \infty} e(k)=A-\Delta r \alpha$

According to the equation (23), the state error is influenced by modeling precision, type of input signals and robust filter parameters.

If the input signal is a step, then $e(\infty)=\lim _{k \rightarrow \infty} e(k)=A$

According to the equations (21), (22) and (23), the robust of the IMC system is enhanced by the larger $\alpha(0<\alpha<1)$.

\section{SIMULATION}

In this paper, MATLAB R2006 is used for the emulation of three-phase active power filter. In the considered power system, the three-phase source has the following characteristics: $L s=2 \mathrm{mH}, V s=220 \mathrm{~V} / 50 \mathrm{~Hz}$. Voltage on DC side is set as $800 \mathrm{~V}$. That is voltage for each capacitor $u d=400 \mathrm{~V}$. The nonlinear load is the three-phase non-controllable bridge rectifier with variable $\mathrm{RL}$ branches $(0 \mathrm{~s}<\mathrm{t}<0.08 \mathrm{~s}, \mathrm{R}=5 \Omega ; 0.08 \mathrm{~s}<\mathrm{t}$ $<0.14 \mathrm{~s}, \mathrm{R}=15 \Omega ; 0.14 \mathrm{~s}<\mathrm{t}, \mathrm{R}=10 \Omega, \mathrm{L}=25 \mathrm{mH})$.

Data Training: In order to have a good stationary and periodicity, the variable RL is replaced by a variable voltage source (a pseudo-random Arms $\in(154,286))$. And the onestep linear filter $F F(z)=0.7 z-1 /(1-0.3 z-1)$. The parameter of $E L M$ is $L=23$. After the data training, the tracking results of square wave of pseudo-linear system and the closed IMC system are shown in Fig. (4). Compared with Fig. (4a) and Fig. (4b), the closed loop system can enhance the output result with modeling error.

Smooth Filtering: In order to test the filter results, if the three voltage sources are harmonic distortion (as shown in Table 1).

With the help of LS-ELM, the IMC system can deal with the different problem as follows: (1) balanced and nondistorted three-phase, (2) $10 \% 5$ th harmonics, (3) $10 \% 7$ th harmonics, (4) unbalanced three-phase voltage with $10 \%$, (5)complex voltage (as Table 1).

Comparing Figs. $(\mathbf{5}, \mathbf{6})$, it can be concluded that V $\alpha$ VS. $\mathrm{V} \beta$ is a round circle under the ideal compensation effect. If the source voltage is more distorted, then V $\alpha \mathrm{VS}$. V $\beta$ is far more from the round circle. In this way, the harmonic suppression result will be weakening. In a complex industrial circumstance, a balanced and non-distorted three-phase voltage is very important. With the help of LS-elm, the referenced ideal three-phase voltage can be obtained. Furthermore, according to the $V \alpha V S$. $V \beta$ dynamic regulation process, smooth filtering is the key function of LS-ELM for harmonic distortion. While the fundamental positive-sequence obtaining is the main function of LS-ELM for unbalanced sources.

Table 1. Distorted voltage parameter

\begin{tabular}{|c|c|c|c|c|c|}
\hline Voltage & fundamental wave & 5th harmonics & 7th harmonics & 11th harmonics & white noise \\
\hline \hline magnitude [p.u.] & $1 \angle 0^{\circ}$ & $0.25 \angle 45^{\circ}$ & $0.25 \angle 180^{\circ}$ & $0.15 \angle 180$ & variance 20 \\
\hline
\end{tabular}



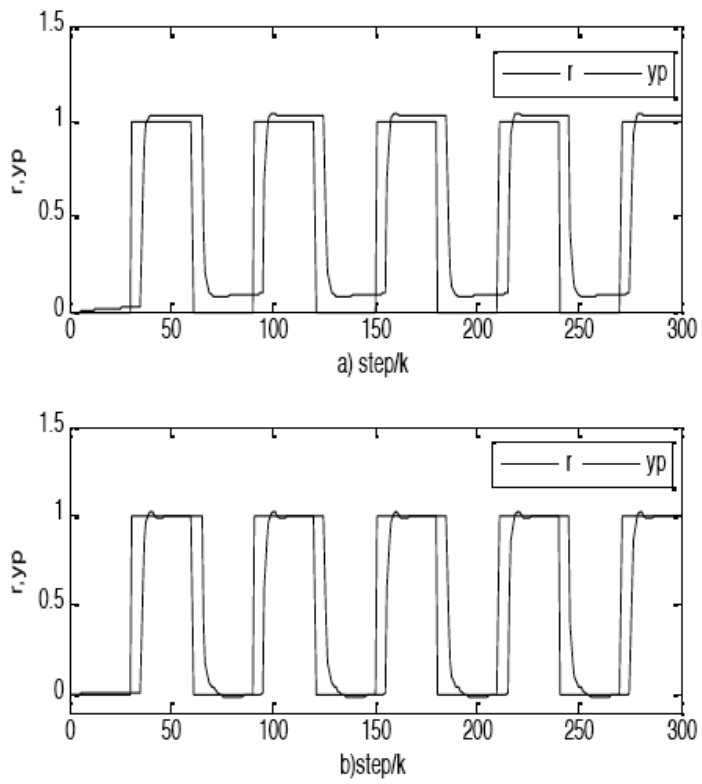

(a) Pseudo-linear open-loop system

(b) Closed IMC system

Fig. (4). Tracking results of square wave.
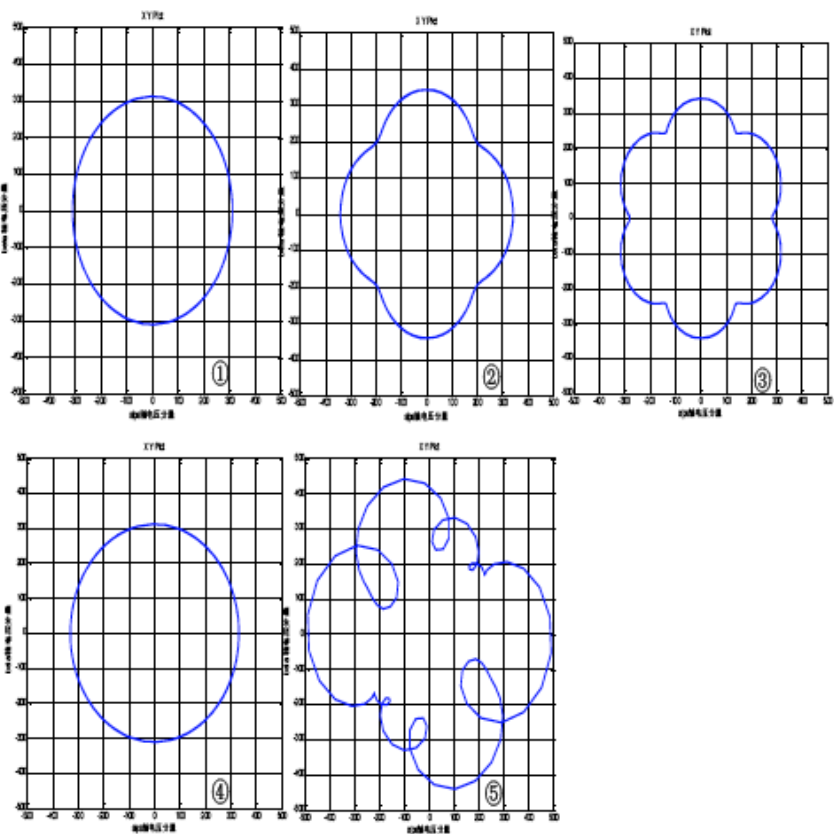

Fig. (5). Different voltage sources, Voltage V $\alpha$ VS V $\beta$ (dq- rotating coordinate system).

\section{CONCLUSION}

In this paper, an internal model controller for three-phase APF based on LS-Extreme Learning Machine is discussed. The simulation results show that the improved elm controller has the advantages of better dynamic performance, being easy to realize and has better tracking performance. And the simulation results also demonstrate the effectiveness of the improved method. On the other hand, the LS-ELM method can meet the requirements of unbalanced and distorted power systems, and has importance and significance in improving the whole performance of APF.
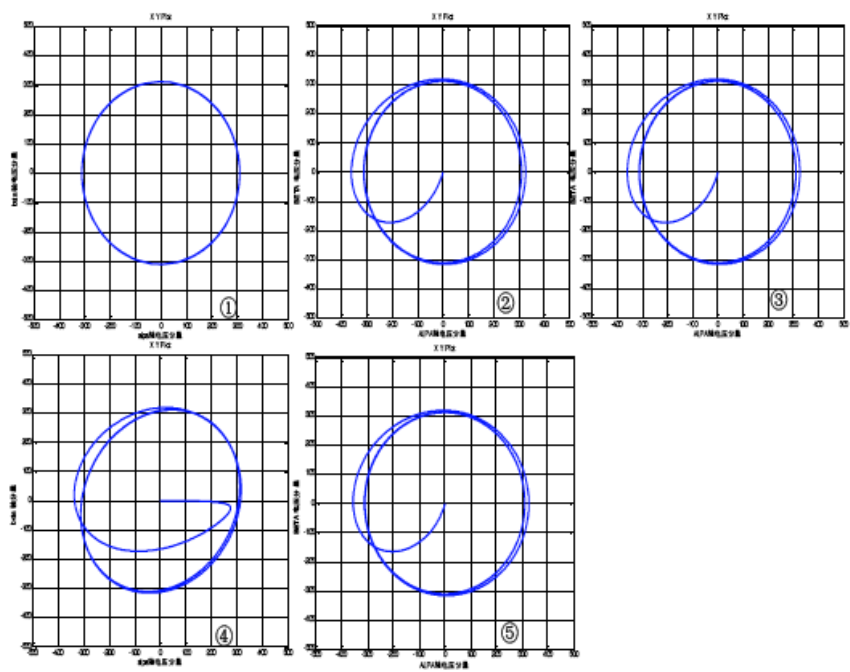

Fig. (6). With LS-ELM, Voltage V $\alpha$ VS. V $\beta$ (dq- rotating coordinate system).

\section{CONFLICT OF INTEREST}

The authors confirm that this article content has no conflict of interest.

\section{ACKNOWLEDGEMENTS}

The Project Supported by Fujian Provincial Major Scientific and Technological Projects under grant 2014H6028, Scientific Research Items of XMUT under grant XYK201401, XMUT Initializing Foundation under grant YKJ12010R, and funding (type A) (funding number: JA12253) from the Fujian Education Department.

\section{REFERENCES}

[1] S. Shukla, S. Mishra, and B. Singh, "Empirical-Mode Decomposition With Hilbert Transform for Power-Quality Assessment," IEEE Transactions on Power Delivery, vol. 24, no. 4, pp. 2159-2165, 2009.

[2] P.E. Sutherland, and T.A. Short, "Power Quality Assessment of Distributed Generator Grounding Method," IEEE Transactions on Industry Applications, vol. 45, no. 1, pp. 303-309, 2009.

[3] M. Karimi-Ghartemani, and M. R. Iravani, "Measurement of harmonics/inter-harmonics of time-varying frequencies," IEEE Transactions on Power Delivery, vol. 20, no.1, pp. 23-31, 2005.

[4] X. B. Bian, and A. D. Bandrauk, "Multichannel Molecular HighOrder Harmonic Generation from Asymmetric Diatomic Molecules," Physical Review Letters, vol. 105, no. 9, 93903, 2010.

[5] N. Bianchi, S. Bolognani, M.D. Pre, and G. Grezzani, "Design considerations for fractional-slot winding configurations of synchronous machines," IEEE Transactions on Industry Applications, vol. 42, no. 4, pp. 997-1006, 2006.

[6] X. B. Bian, A. D. Bandrauk, "Multichannel Molecular High-Order Harmonic Generation from Asymmetric Diatomic Molecules," Physical Review Letters, vol. 105, no. 9, 93903. 2010.

[7] J. Eloy-Garcia, J.C. Vasquez, and J.M. Guerrero, "Grid simulator for power quality assessment of micro-grids," IET Power Electronics, vol. 6, no. 4, pp. 700-709, 2013.

[8] X. Wang, J. Yong, W. Xu, and W. Freitas, "Practical Power Quality Charts for Motor Starting Assessment," IEEE Transactions on Power Delivery, vol. 26, no. 2, pp. 799-808, 2011.

[9] S. Zhang, K.J. Tseng, and S.S. Choi, "Statistical voltage quality assessment method for grids with wind power generation," IET Renewable Power Generation, vol. 4, no. 1, pp. 43-54, 2010.

[10] L. Marconi, F. Ronchi, and A. Tilli, "Robust nonlinear control of shunt active filters for harmonic current compensation," Automatica, vol. 43, pp. 252-263, 2007. 
[11] G.B. Huang, Q.Y. Zhu, C.K. Siew, "Extreme learning machine: Theory and applications," Neurocomputing, vol. 70, pp. 489-501, 2006

[12] G.B. Huang, C.K. Siew, "Extreme learning machine: RBF network case," In: Proceedings of the $8^{\text {th }}$ International Conference on Control, Automation, Robotics and Vision, 2004, China, Kunming.

[13] Q.Y. Zhu, A.K. Qin, P.N. Suganthan, and G.B. Huang, "Evolutionary extreme learning machine," Pattern Recognition, vol. 38, no. 2, pp. $1759-1763,2005$
[14] M. Cirrincione, M. Pucci, and G. Vitale, "Current Harmonic Compensation by a Single-Phase Shunt Active Power Filter Controlled by Adaptive Neural Filtering," IEEE Transactions on Industrial Electronics, vol. 56, no. 8, pp. 3128-3142, 2009

[15] G.W. Chang, C. Chen, and Y.F. Teng, "Radial-Basis-FunctionBased Neural Network for Harmonic Detection," IEEE Trans. On Industrial Electronics, vol. 57, no. 6, pp. 2171-2179, 2010.

Received: October 16, 2014

Revised: November 24, 2014

Accepted: November 28, 2014

(C) Shao et al.; Licensee Bentham Open.

This is an open access article licensed under the terms of the (https://creativecommons.org/licenses/by/4.0/legalcode), which permits unrestricted, noncommercial use, distribution and reproduction in any medium, provided the work is properly cited. 